\title{
Dynamic failure of reinforced concrete plates
}

\author{
H. Sjøl
}

Norwegian Defence Research Establishment, P.O.Box 25, 2007 Kjeller, Norway

\begin{abstract}
Résumé
Une étude théorique en ce qui concerne les plaques rectangulaires exposées à un soufle d'air est vérifié par la théorie de Lagrange pour les petites deflexions des plaques minces, de. la théorie de von Karman pour de grandes deflexions de plaques minces et de la théorie de Mindlin pour des plaques relativement épaissés. Les théories des plaques sont analysées pour approximer les murs et les plafonds d'une structure complexe. Toutes les modélisations sont faites à partier de modéles de materiaux isotropes. Les diagrammes de Pression-Impulsion sont construits pour prévoir la charge à la rupture. Les charges critiques, qui sont à l'origine des dégâts, sont déterminées pour les plaques rectangulaires en fonction du rapport de l'épaisseur sur l'envergure. Les résultats numériques d'une structure "boite" sont calculés pour verifier l'approximation des plaques. Plusieurs résultats experimentaux sont aussi decrits.
\end{abstract}

\begin{abstract}
A theoretical study of rectangular plates exposed to air blast load is performed using Lagrange's theory for small deflections of thin plates, von Karman's theory for large deflections of thin plates and Mindlin's theory for (moderately) thick plates. The plate theories are analysed to approximate the walls and roofs of a complex structure. All modelling are done with isotropic material models. Pressure-Impulse diagrams (PI-diagrams) are constructed to predict the flexural damage load.

Critical loads, which initiate damage, are determined for rectangular plates as a function of thickness to span ratio. Numerical results of a box structure are presented to check the plate approximation of the wall. Some experimental results are also described.
\end{abstract}

\section{INTRODUCTION}

All structures built for military purposes, are designed to resist a certain load. Such structures can for example be protective structures made of reinforced concrete, fighting ships or vehicles. These structures may, in wartime, be exposed to hostile actions. Different kinds of weapon can be used against the targets depending on the military value of the structure and the necessary effort to cause severe structural damage. Hence, in general one has to study all potential weapon 
effects, such as penetration, air blast, fragments and ground shock, against each structure. In the following, only air blast load will be studied. The roof of a box structure is exposed to an air blast load, approximated by

$$
p(t)=P e^{-t / T}
$$

where $P$ is the peak pressure, and $T$ can be regarded as the duration of the load. A schematic graph of the pressure-time relationship is shown in figure 1.

Instead of the duration, the load can, in addition to the the peak pressure, be described by its total impulse, defined by

$$
I=\int_{0}^{\infty} p(t) d t
$$

In the particular case described in this paper, $I=P T$. Damage curves for structures exposed to air blast load, can be drawn in Pressure-Impulse diagrams (PI-diagrams), as shown in figure 1. Such curves give combinations of peak pressure and total impulse which give identical damage levels. The load is called impulsive if the duration $T$ is much less than the natural period of the exposed structure, and quasi-static if the duration $T$ is much larger than the natural period. Pressure and impulse above the curve cause damage to the structure, while combinations of pressure and imulse below the curve are not sufficient to cause damage to the structure. The asymptotes are independent of the pressure-time relationship. Only the "curved" part of the PI-diagram depends on the shape of the pressure-time-curve. In the present paper, only the asymptotes will be analysed.
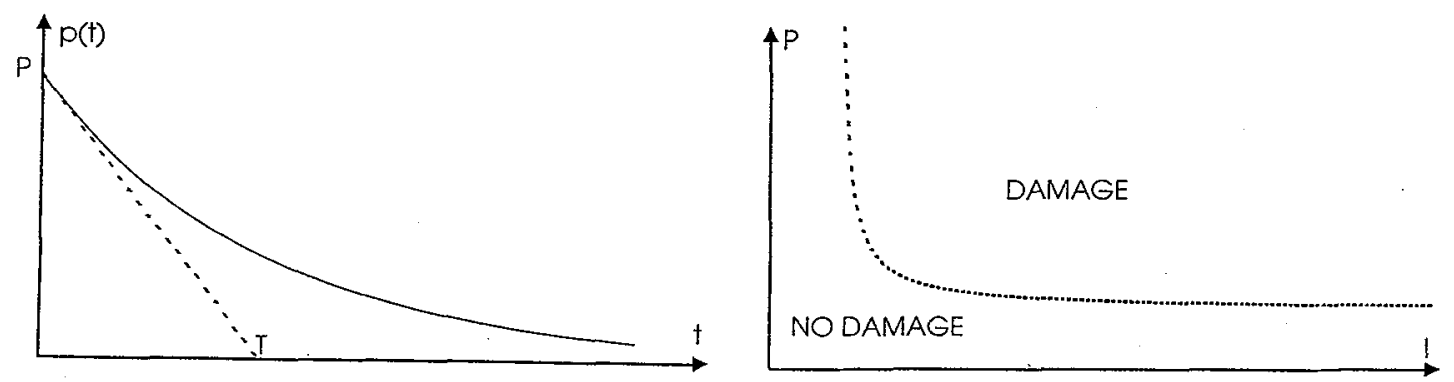

Figure 1: a) The pressure-time-relationship used in the present paper b) A general PI-diagram

A theoretical approach to describe the damage of a complex structure is here done by analysing the walls and roofs in the structures as rectangular plates. The supporting walls are the boundary conditions, but for simplicity, the plates analysed will either be simply supported or clamped. It is natural to assume that the damage curves for the exposed wall in a complex structure lies between the damage curves for simply supported and clamped plates.

Several plate theories are studied to approximate the damage level of the roof of the boxstructure. In addition to the classical plate theory (Lagrange (1811)[1]), the simplest plate theories taking large deformations (von Karman (1910)[1]) and shear deformations (Reissner(1945)[2] / Mindlin(1951)[3]), respectively, are analysed. 
An introduction to PI-diagrams for simple mechanical systems, such as harmonic oscillators, can be found in Abrahamson \& Lindberg [4]. Since the plate equations can be reduced to a harmonic oscillator when studying small deflections of plates, PI-diagrams are suitable to present dynamic responce of plates.

Ari-Gur \& Kenig (1990) [5] presented iso-deflection curves in the PI-plane using Lagrange's plate theory for orthotropic plates with an exact solution' (infinite Fourier series) for the out-ofplane deflection. Here no damage criterion is implemented, but the approach of Ari-Gur \& Kenig can easily be extended to include a critical displacement found from von Mises yield criterion.

The aim of the present work is to compare the the loads which initiate damage to rectangular plates found from several plate theories. Damage curves in the PI-plane for both thin and thick plates are presented. This is an extension of the curves presented by Ari-Gur \& Kenig [5] in the sense that both large geometrical deformations and transverse shear deformations are taken into account. These theoretical damage curves are compared to the damage curves for the roof of a box structure, to validate the approximation of walls and roofs in a complex structure by rectangular plates. The theoretical calculations are done with an isotropic material model, but are compared to some experimental results on reinforced concrete plates.

\section{GOVERNING EQUATIONS}

The plates studied in this paper are exposed to a uniform step pulse loading with an exponential decay on the top surface, given in equation (1).

In all three plate theories, the displacement field is assumed to be on the form $u=z \psi_{1}(x, y, t)$, $v=z \psi_{2}(x, y, t)$ and $w=\zeta(x, y, t)$, where $u, v$ and $w$ are the displacement of the plare in the $x^{-}$, $y$ - and $z$-direction, respectively. The $z$-axis is directed normal to the plate surface. In the theories for thin plates, the functions $\psi_{1}$ and $\psi_{2}$ are approximated by $\psi_{1}=-\frac{\partial \zeta}{\partial x}$ and $\psi_{2}=-\frac{\partial \zeta}{\partial y}$, giving only one unknown displacement parameter.

The boundary conditions studied in the present paper, are either "simply supported" ( $\zeta=$ $\frac{\partial^{2} \zeta}{\partial n^{2}}=0$ at the boundary) or "clamped" ( $\zeta=\frac{\partial \zeta}{\partial n}=0$ at the boundary). The following notation is used is the governing equations to simplify the writing of the equations: $\nabla^{4}=\frac{\partial^{4}}{\partial x^{4}}+2 \frac{\partial^{4}}{\partial x^{2} \partial y^{2}}+\frac{\partial^{4}}{\partial y^{4}}$ and $\ddot{O}=\frac{\partial^{2}}{\partial t^{2}}$. The out-of-plane deflection governed by linear thin plate theory [1] is found from

$$
D \nabla^{4} \zeta+\rho h \ddot{\zeta}=p(t)
$$

where $D=\frac{E h^{3}}{12\left(1-\nu^{2}\right)}, E$ is the modulus of elasticity, $\rho$ is the density of the plate, $h$ is the plate thickness and $\nu$ is the Poisson's ratio.

When including the effects of non-linear geometrical deformations, the out-of-plane deflection for thin plates is found from the coupled equations [1]

$$
\begin{aligned}
D \nabla^{4} \zeta-h\left(\frac{\partial^{2} \chi}{\partial y^{2}} \frac{\partial^{2} \zeta}{\partial x^{2}}+\frac{\partial^{2} \chi}{\partial x^{2}} \frac{\partial^{2} \zeta}{\partial y^{2}}-2 \frac{\partial^{2} \chi}{\partial x \partial y} \frac{\partial^{2} \zeta}{\partial x \partial y}\right)+\rho h \ddot{\zeta} & =p(t) \\
D \nabla^{4} \chi+E\left(\frac{\partial^{2} \zeta}{\partial x^{2}} \frac{\partial^{2} \zeta}{\partial y^{2}}-\left(\frac{\partial^{2} \zeta}{\partial x \partial y}\right)^{2}\right) & =0
\end{aligned}
$$

where $\chi$ is Airy's stress function, defined by $\sigma_{x x}=\frac{\partial^{2} \chi}{\partial y^{2}}, \sigma_{y y}=\frac{\partial^{2} \chi}{\partial x^{2}}$ and $\sigma_{x y}=-\frac{\partial^{2} \chi}{\partial x \partial y}$.

The displacement components in thick plate theory [3] are found from the following equations:

$$
\begin{aligned}
\frac{D}{2}\left((1-\nu) \nabla^{2} \psi_{1}+(1+\nu) \frac{\partial \Phi}{\partial x}\right)-\kappa^{2} G h\left(\psi_{1}+\frac{\partial \zeta_{M}}{\partial x}\right) & =\frac{\rho h^{3}}{12} \ddot{\psi_{1}} \\
\frac{D}{2}\left((1-\nu) \nabla^{2} \psi_{2}+(1+\nu) \frac{\partial \Phi}{\partial y}\right)-\kappa^{2} G h\left(\psi_{2}+\frac{\partial \zeta_{M}}{\partial y}\right) & =\frac{\rho h^{3}}{12} \ddot{\psi_{2}} \\
\kappa^{2} G h\left(\nabla^{2} \zeta_{M}+\Phi\right)+p(t) & =\rho h \ddot{\zeta_{M}}
\end{aligned}
$$


where $\Phi=\psi_{1, x}+\psi_{2, y}, G=\frac{E}{2(1+\nu)}$ and $\kappa^{2}=\frac{5}{6}$.

\section{FAILURE MODES}

A good failure model of a reinforced concrete structure is dependent on the nature of the applied load. Flexural failure due to large stress values through the structure is one possible failure mode. Shear failure is due to large shear stresses, normally along the connection between two structural parts. Spalling and breaching can occur when the tensile strength is much less than the compressive strenth, which indeed is true for concrete. Normally, a combination of these three failure modes will be present. Hence, it is important to understand all possible failure mechanisms, and in which regimes of the load parameters the different damage mechanisms are initiated. In the present paper, flexural failure will be analysed. Due to the brittle behaviour of concrete, only elastic deformations are studied, and the damage level is given by von Mises yield criterion:

$$
\sigma_{x x}^{2}+\sigma_{y y}^{2}-\sigma_{x x} \sigma_{y y}+3\left(\sigma_{x y}^{2}+\sigma_{y z}^{2}+\sigma_{z x}^{2}\right)=\sigma_{Y}^{2}
$$

where $\sigma_{\alpha \beta}$ are the stresses in the plate, and $\sigma_{Y}$ is the yield stress in uniaxial tension.

\section{DAMAGE CURVES}

The critical quasi-static pressure and critical impulse are found solving the plate equations in connection with von Mises yield criterion. The elastic solution of the plate equations can be written as general Fourier series :

$$
\begin{aligned}
\psi_{1}(x, y, t) & =\Sigma \Sigma A_{i j}(t) \psi_{1 i j}(x, y) \\
\psi_{2}(x, y, t) & =\Sigma \Sigma B_{i j}(t) \psi_{2 i j}(x, y) \\
\zeta(x, y, t) & =h \Sigma \Sigma C_{i j}(t) \psi_{3 i j}(x, y)
\end{aligned}
$$

where $\psi_{k i j}(k=1,2,3)$ satisfies the boundary conditions. Galerkin's method is used to determine the unknown parameters $A_{i j}, B_{i j}$ and $C_{i j}$. To analyse the critical load, the following dimensionless quantities are used :

$$
\begin{aligned}
& \bar{P}_{\text {crit }}=\frac{P_{c r i t}}{\sigma_{Y}} \frac{a}{h} \\
& \bar{I}_{c r i t}=\frac{I_{c r i t}}{\sigma_{Y} h \sqrt{\rho / E}}
\end{aligned}
$$

where $P_{\text {crit }}$ and $I_{c r i t}$ are the quasi-static pressure and total impulse, respectively, giving a state of stress which satisfy equation (9) and the plate equations. The quantity a denotes the span of the plate, and the other quantities are defined in section 2 . The critical load parameters as a function of the thickness to span ratio are shown in figure 2. Numerical results from the box-simulations are also shown in figure 2. These numerical simulations are performed with the finite element code DYNA3D [6], and the simulation parameters are: The roof (the exposed surface) has outer dimensions of $2 \mathrm{~m} \mathrm{x} 2 \mathrm{~m}$ with a thickness varying from $0.1 \mathrm{~m}$ to $0.2 \mathrm{~m}$. The total height of the box is varying from $0.5 \mathrm{~m}$ to $0.6 \mathrm{~m}$, and the wall thickness is $0.4 \mathrm{~m}$. The roof has an internal span of $1.2 \mathrm{~m}$. To determine whether the external or internal dimensions of the roof shall be used, both possibilities are drawn into figure 2 .

Some experiments have been performed at the Norwegian Defence Research Establishment (NDRE) with bare sperical explosive charges (charge weight $<50$ grams) on a simply supported 
reinforced concrete plate with the following parameters: $a=0.30 \mathrm{~m}, h=0.03 \mathrm{~m}(h / a=0.10)$, $E=33 \mathrm{GPa}, \nu=0.2$ and $f_{c}=35 \mathrm{MPa}$, where $f_{c}$ is the uniaxial compressive strength of concrete. The distance from the charge to the plate varied between 150 and $350 \mathrm{~mm}$, which indicate that the shock front can not be regarded as plane, and that the arrival time of the shock front is significantly different at the edges than in the centre of the plate. I.e., the critical impulse found from the experiments will be overestimated compared to an "equivalent plane impulse". The scaled impulse initiating small cracks in the concrete plates is found to be approximately 0.50 , while the theoretical value is 0.35 (The yield stress $\sigma_{Y}$ is replaced by the compressive strength $f_{c}$ in computing $\bar{I}_{\text {crit }}$ in equation (11)). The "equivalent plane impulse" is the plane impulse giving almost identical damage to the structure as the spherical load produced by the explosive charge. When the distance from detonation to the plate is of the same size as half the span of the plate, a reduction factor to obtain an "equivalent plane impulse" is approximately 0.6 . This gives an equivalent critical plane impulse of 0.30 , which is close to the theoretical value of 0.35 .
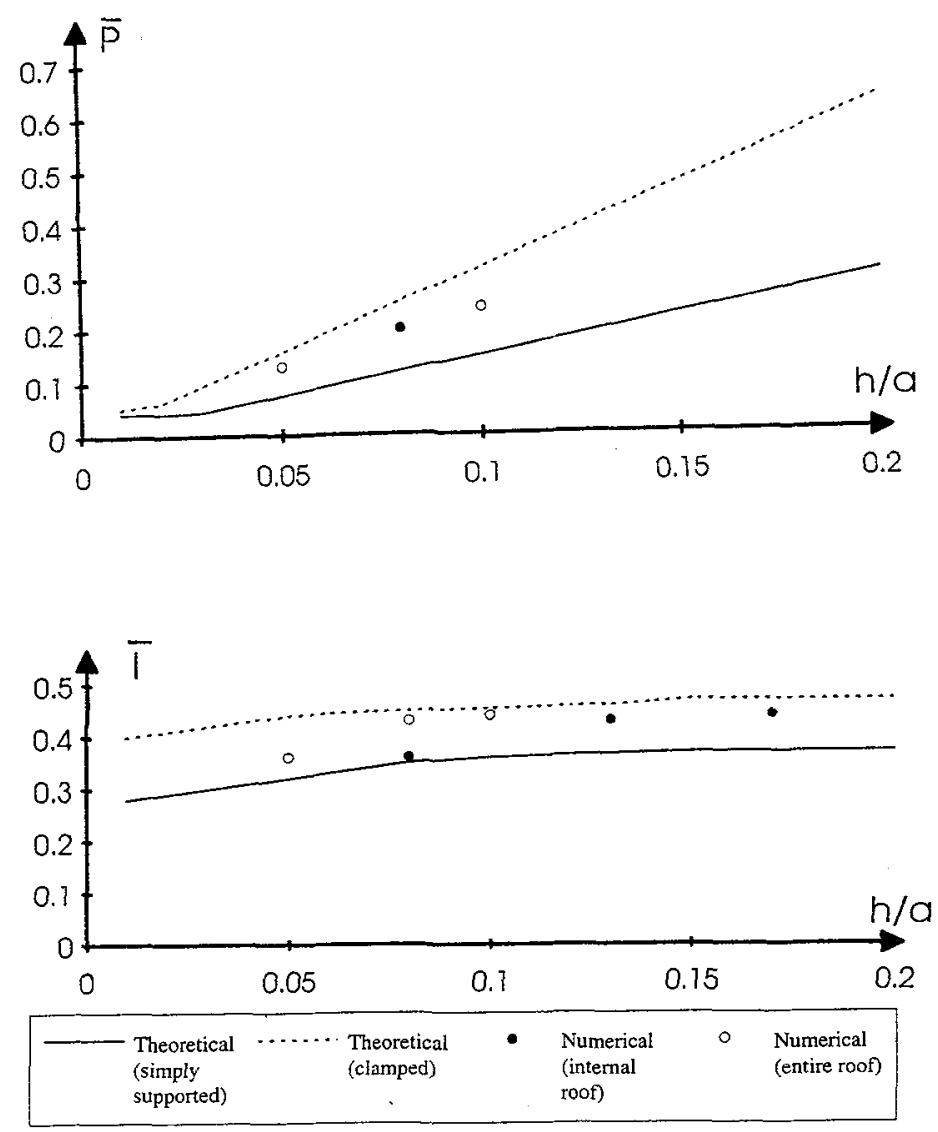

Figure 2: a) The critical quasi-static pressure and b) the critical impulse as a function of the thickness to span ratio 


\section{DISCUSSION AND CONCLUSION}

Large deformations of the plate only come into consideration when analysing thin plates (thickness to span ratio less than 0.03 ). Transverse shear deformations are significant when determining the elastic deformations of thick plates (thickness to span ratio larger than 0.10 ), but this effect is almost neglible when determining the loads which initiate damage to a rectangular plate. I.e., thin plate theories give acceptable results also for thick plates. For very thick plates (thickness to span ratio about 0.20 ), however, the assumptions in the displacement field are no longer valid, and a more sophisticated theoretical model may give more accurate results.

As indicated in section 1, the damage curves for the roof, when treated as a rectangular plate, is somewhere between the damage curves for simply supported and clamped plates. The results seem to fit both the internal and external roof dimensions. For impulsive loading, the thick roofs behave like a clamped plate, while the numerical results for thinner roofs are close to the theoretical values for simply supported plates. For thick roofs, the rotations of the roof in the connection between the roof and the walls are neglible, which indicates results close to a clamped plate, while for thinner roofs, this rotation is significant, and the damage curves for a thin roof in a complex structure are close to the damage curves for a simply supported plate with the same dimensions as the roof. For quasi-static loads, this trend is, however, not visible from the present analysis.

The roof or walls in a complex structure can be treated as a rectangular plate when determining flexural failure, as shown in figure 2. The theoretical results for simply supported and clamped plates form lower and upper bounds for the critical load parameters of the roof of a box-structure, respectively.

The experiments in the impulsive domain shows that an isotropic material model can be used for reinforced concrete when calculating the impulse which initiates damage.

A more detailed analysis of PI-diagrams for rectangular plates can be found in [7].

\section{References}

[1] Landau, L.D. \& Lifshitz, E.M. Theory of elasticity 2. edition, Pergamon Press (1970)

[2] Reissner, E. The effect of transverse shear deformation of elastic plates Journal of Applied Mechanics, 12 (1945), pp 69-77

[3] Mindlin, R.D. Influence of rotatory inertia and shear on flexural motions of isotropic, elastic plates Journal of applied mechanics (1951), 18, pp 31-38

[4] Abrahamson, G.R. \& Lindberg, H.E. Peak load-impulse characterization of critical pulse loads in structural mechanics Nuclear engineering and design, 37 (1976), pp 35-46

[5] Ari-Gur, J. \& Kenig, M. J. Unified iso-deflection curves for orthotropic plates under lateral pressure pulse Journal of applied mechanics, 57 (1990), pp 470-472

[6] Hallquist, J.O. \& Brown, B. E. DYNA3D user's manual Lawrence Livermore National Laboratory (1984)

[7] Sjøl, H. Damage criteria for rectangular plates exposed to lateral pressure loadings. Dr. Scient thesis, University of Oslo (1994) 